\title{
Billiards and Tilting Characters for $\mathrm{SL}_{3}$
}

George LUSZTIG ${ }^{\dagger}$ and Geordie WILLIAMSON ${ }^{\ddagger}$

$\dagger$ Massachusetts Institute of Technology, Cambridge, MA, USA

E-mail: gyuri@math.mit.edu

URL: http://www-math.mit.edu/ gyuri/

$\ddagger$ Sydney University, Sydney, NSW, Australia

E-mail: g.williamson@sydney.edu.au

URL: http://www.maths.usyd.edu.au/u/geordie/

Received July 18, 2017, in final form February 16, 2018; Published online February 21, 2018

https://doi.org/10.3842/SIGMA.2018.015

\begin{abstract}
We formulate a conjecture for the second generation characters of indecomposable tilting modules for $\mathrm{SL}_{3}$. This gives many new conjectural decomposition numbers for symmetric groups. Our conjecture can be interpreted as saying that these characters are governed by a discrete dynamical system ("billiards bouncing in alcoves"). The conjecture implies that decomposition numbers for symmetric groups display (at least) exponential growth.
\end{abstract}

Key words: tilting modules; billiards; $p$-canonical basis; symmetric group

2010 Mathematics Subject Classification: 20C20; 17B10; 20C30

\section{Introduction}

We formulate a conjecture for the second generation characters of indecomposable tilting modules for $\mathrm{SL}_{3}$ in characteristic $p>2$. These conjectures resulted from our attempts to understand data [25] obtained following a 9 month calculation in magma on a supercomputer at the MPIM in Bonn. These results go far beyond existing calculations and are obtained using a new algorithm [26]. The algorithm relies in an essential way on ideas of Libedinsky, Riche and the second author (see $[15,21])$. The behaviour we observe appears highly non-trivial, which suggests that proving anything might be difficult.

On the next page the reader will find a picture. This picture was obtained by analyzing the output of computer calculations for $p=5$. Exactly how this picture is used to produce (second generation) tilting characters will be explained in the final section. Before reading the rest of the paper the reader is invited to consider this picture and try to discern any patterns. This paper is an attempt to explain this picture, as well as similar pictures for $p=3$ and 7 .

\section{Generational philosophy}

Let $G$ denote a split simple and simply connected algebraic group over a field $\mathbb{k}$ of characteristic $p$. We fix a Borel subgroup and maximal torus $T \subset B \subset G$. We will try to follow the notation of [24]. In particular:

This paper is a contribution to the Special Issue on the Representation Theory of the Symmetric Groups and Related Topics. The full collection is available at https://www.emis.de/journals/SIGMA/symmetric-groups2018.html 


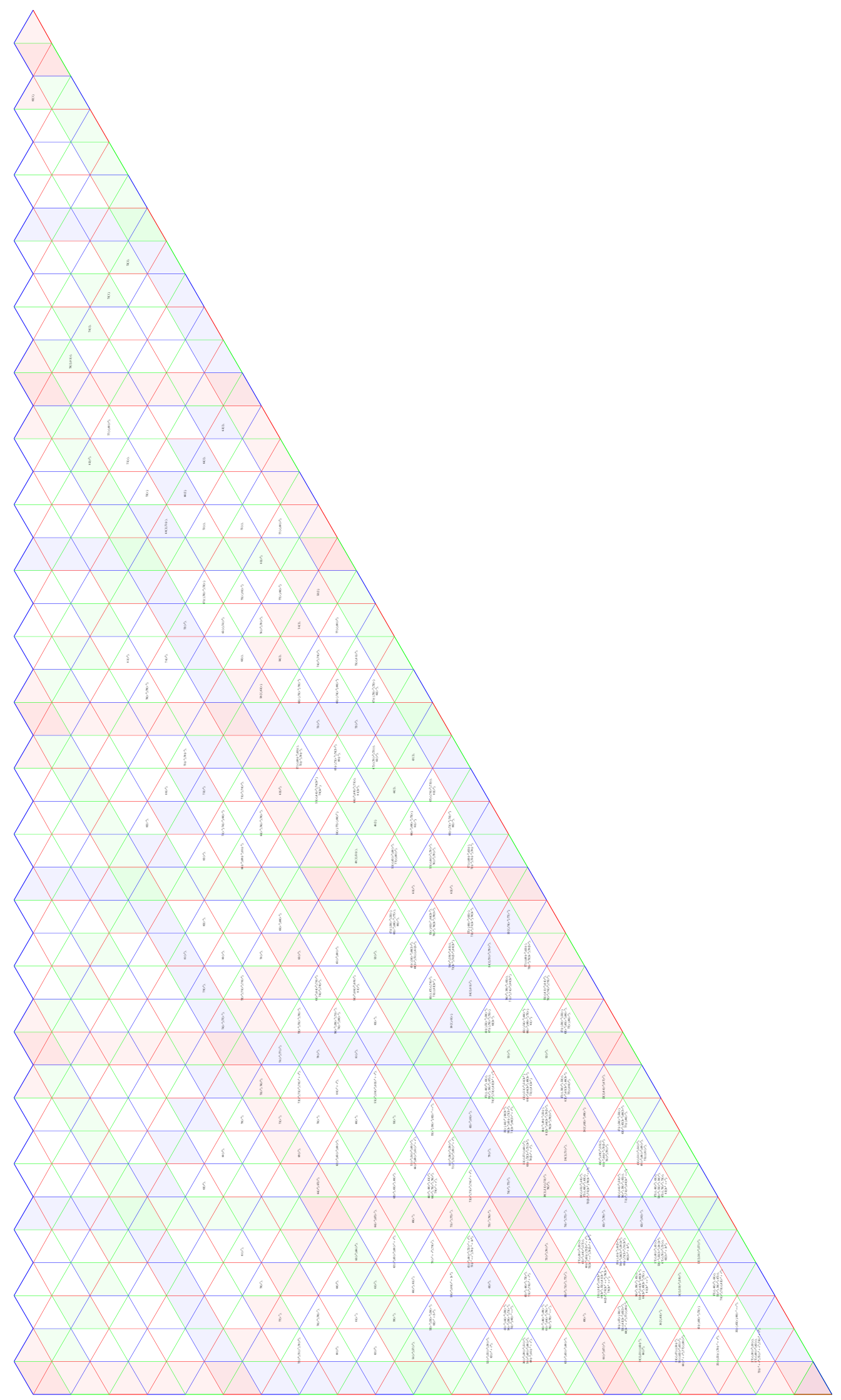

Figure 1. Second generation pattern for $p=5$ up to $i=81$. 


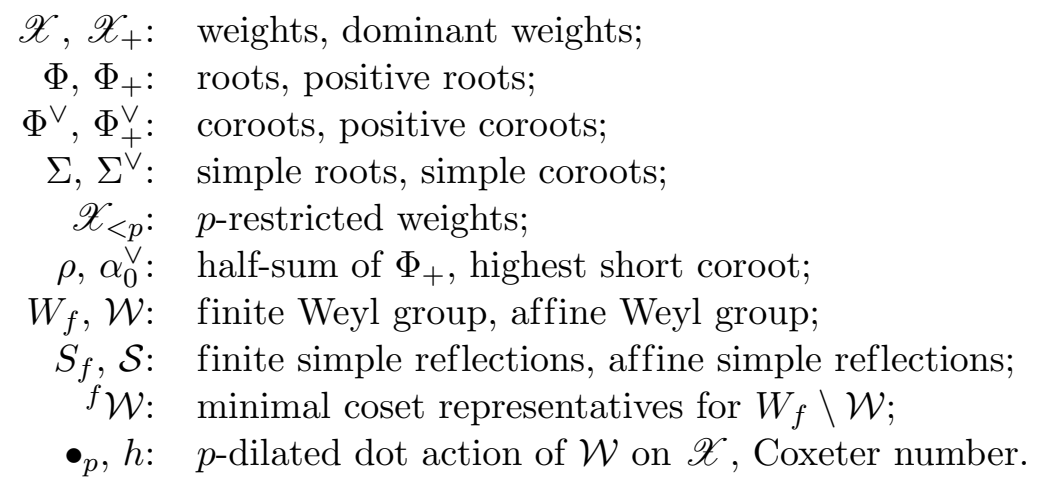

For every dominant weight $\lambda \in \mathscr{X}_{+}$, let $L_{\lambda}$ (resp. $\Delta_{\lambda}$, resp. $T_{\lambda}$ ) denote the simple (resp. standard, resp. indecomposable tilting) module with highest weight $\lambda$.

\subsection{Generations for simple characters}

For fixed $p$ and any highest weight $\lambda \in \mathscr{X}_{+}$the first author has recently defined characters [17]

$$
\chi_{\lambda}^{0}, \chi_{\lambda}^{1}, \chi_{\lambda}^{2}, \ldots, \chi_{\lambda}^{\infty} \in(\mathbb{Z} \mathscr{X})^{W_{f}}
$$

with the following properties:

1) $\chi_{\lambda}^{0}$ is the character of the simple highest weight module in characteristic 0 (i.e., $\chi_{\lambda}^{0}$ is given by Weyl's character formula);

2) $\chi_{\lambda}^{1}$ is the character of the simple highest weight module for the quantum group at a $p^{\text {th }}$-root of unity;

3) $\chi_{\lambda}^{n}$ is obtained from $\chi_{\lambda}^{n-1}$ by a formula involving Kazhdan-Lusztig polynomials;

4) if $\lambda=\lambda_{0}+p \lambda_{1}+\cdots+p^{n} \lambda_{n}$ with $\lambda_{i} \in \mathscr{X}_{<p}$ and $\left\langle\alpha_{0}^{\vee}, \lambda_{n}+\rho\right\rangle \leq p$ (a generalised Jantzen condition) then $\chi_{\lambda}^{n}=\chi_{\lambda}^{n+1}=\cdots=\chi_{\lambda}^{\infty}$;

5) if $p$ is large then $\chi_{\lambda}^{\infty}$ is the character of $L_{\lambda}$.

Remark 2.1. Recall that the Steinberg tensor product theorem has "one step" for the quantum group at a root of unity, and "infinitely many steps" for $G$. The characters $\chi_{\lambda}^{n}$ can be thought of as the simple characters for an object with an " $n$ step" Steinberg tensor product theorem. It is not known whether such an object exists. For $\mathfrak{s l}_{2}$ such an object (for any $n$ ) has recently been proposed by Angiono [6]. It seems likely that one can combine recent work of Elias [8] with work of Riche and the second author [21] to construct such an object in type $A$ for $n=2$.

\subsection{Generations for tilting characters}

As for simple characters, there should exist approximations to tilting characters. That is, for any $p$ and dominant weight $\lambda \in \mathscr{X}$ there should exist characters

$$
\theta_{\lambda}^{0}, \theta_{\lambda}^{1}, \theta_{\lambda}^{2}, \ldots, \theta_{\lambda}^{\infty} \in(\mathbb{Z} \mathscr{X})^{W_{f}}
$$

with the following properties:

1) $\theta_{\lambda}^{0}$ is the character of the simple highest weight module in characteristic 0 (i.e., $\theta_{\lambda}^{0}$ is given by Weyl's character formula);

2) $\theta_{\lambda}^{1}$ is the character of the indecomposable tilting module for the quantum group at a $p^{\text {th }}$ root of unity; 
3) $\theta_{\lambda}^{n}$ is a positive linear combination of the $\theta_{\mu}^{n-1}$ 's for all $n \geq 1$;

4) if $\left\langle\alpha_{0}^{\vee}, \lambda+\rho\right\rangle \leq p^{n+1}$ then $\theta_{\lambda}^{n}=\theta_{\lambda}^{n+1}=\cdots=\theta_{\lambda}^{\infty}$;

5) if $p$ is large then $\theta_{\lambda}^{\infty}$ is the character of $T_{\lambda}$.

Remark 2.2. Parts (4) and (5) for $n=1$ imply Andersen's conjecture [5], which is still open (even for $p$ large).

Remark 2.3. The characters $\theta_{\lambda}^{n}$ are defined for a subset of $\mathscr{X}_{+}$(roughly, those for which one can apply the tilting tensor product theorem) in [18].

\subsection{Our conjecture at $v=1$}

Let us assume that $p \geq h$, and consider the $p$-dilated action of the affine Weyl group from above. For any choice of "generation parameters" $0 \leq m \leq n \leq \infty$ we can write

$$
\theta_{x \bullet_{p} 0}^{n}=\sum_{y \in W} d_{y, x}^{m, n} \cdot \theta_{y \bullet_{p} 0}^{m}
$$

for some $d_{y, x}^{m, n} \in \mathbb{Z}_{\geq 0}$. (The fact that we can form such expressions follows from the linkage principle and our assumptions above.) The question of determining characters of indecomposable tilting characters is equivalent to determining the coefficients $d_{y, x}^{\infty, 0}$ for all $y, x \in{ }^{f} W$.

Remark 2.4. A philosophy underlying the current paper is that it might be easier to calculate the $d_{y, x}^{n+1, n}$ for all $n$, rather than calculate the $d_{y, x}^{\infty, 0}$ directly. This is the case for simple characters, as explained in Section 2.1.

The following is known about these coefficients:

1) when $G=\mathrm{GL}_{n}$, the $d_{y, x}^{\infty, 0}$ are equal to decomposition numbers for symmetric groups, by work of Donkin [7] and Erdmann [10];

2) the $d_{y, x}^{1,0}$ are given as the value at 1 of certain parabolic Kazhdan-Lusztig polynomials, by work of Soergel [22, 23];

3) the $d_{y, x}^{\infty, 1}$ are equal to coefficients of James' "adjustment matrix" [11].

Remark 2.5. Aside from the above, very little is known. The papers $[12,20]$ contain some interesting calculations for $\mathrm{SL}_{3}$

In this paper we formulate a conjecture for ( $v$-analogues of the) coefficients $d_{y, x}^{2,1}$, when $G=\mathrm{SL}_{3}$. (Note that in the case of $G=\mathrm{SL}_{3}$ the coefficients $d_{y, x}^{1,0}$ given by Soergel's algorithm are easily calculated, and may be described by closed formulas.) By property (4) of the previous section, this provides a conjecture for many $d_{y, x}^{\infty, 1}$, and hence for many coefficients of the adjustment matrix (and hence decomposition numbers) for three row partitions.

\subsection{Generations for the $p$-canonical basis}

Let $\mathrm{H}$ denote the Iwahori-Hecke algebra of the affine Weyl group $\mathcal{W}$ over $\mathbb{Z}\left[v^{ \pm 1}\right]$. Consider the anti-spherical module (see, e.g., [21, 24]):

$$
\mathrm{AS}_{v}:=\operatorname{sgn}_{v} \otimes_{\mathrm{H}_{f}} \mathrm{H}=\bigoplus_{x \in^{f} \mathcal{W}} \mathbb{Z}\left[v^{ \pm 1}\right] n_{x} .
$$

The anti-spherical module has a canonical basis $\left\{\underline{n}_{x} \mid x \in{ }^{f} \mathcal{W}\right\}$ and a $p$-canonical basis $\left\{{ }^{p} \underline{n}_{x} \mid x \in\right.$ $\left.{ }^{f} \mathcal{W}\right\}[14,21]$. 
In [21] (see also [24, Section 2.8]) it is conjectured that if we write

$$
{ }^{p} \underline{n}_{x}=\sum{ }^{p} m_{y, x} n_{y}
$$

then the values at 1 of the coefficients ${ }^{p} m_{y, x}$ express the characters of tilting modules in terms of Weyl characters. ${ }^{1}$ Let us give a precise version of this conjecture for $p \geq h$ : for all $x, y \in{ }^{f} \mathcal{W}$ we have (in the notation of Section 2.3)

$$
d_{y, x}^{\infty, 0}={ }^{p} m_{y, x}(1) .
$$

(Recall that, by the translation principle and still under our assumption $p \geq h$, knowledge of the left hand side of (2.2) for all $x, y \in{ }^{f} \mathcal{W}$ implies knowledge of the characters of all tilting modules for $G$.)

Remark 2.6. In [21] this conjecture is proved for $\mathrm{GL}_{n}$ and $p>h$. In [9] this conjecture is proved for $\mathrm{GL}_{n}$ for general $p$. In $[1,2,4]$ this conjecture is proved in all types for $p>h$.

The presence of $v$ is the shadow of a non-trivial grading on the category of tilting modules. ${ }^{2}$ It is natural to expect that the above approximations to tilting characters can also be made to respect this grading. That is, we expect that for all $p>h$ and $x \in{ }^{f} \mathcal{W}$, there exist elements

$$
{ }^{p} \underline{n}_{x}^{1},{ }^{p} \underline{n}_{x}^{2}, \ldots,{ }^{p} \underline{n}_{x}^{\infty} \in \mathrm{AS}_{v}
$$

such that:

1) ${ }^{p} \underline{n}_{x}^{1}=\underline{n}_{x}$

2) ${ }^{p} \underline{n}_{x}^{n}$ is a $\mathbb{Z}_{\geq 0}\left[v^{ \pm 1}\right]$-linear combination of the ${ }^{p} \underline{n}_{y}^{n-1}$ 's for all $n \geq 1$;

3) if $\left\langle\alpha_{0}^{\vee}, x \bullet{ }_{p} 0+\rho\right\rangle \leq p^{n+1}$ then ${ }^{p} \underline{n}_{x}^{n}={ }^{p} \underline{n}_{x}^{n+1}=\cdots={ }^{p} \underline{n}_{x}^{\infty}$;

4) if $p$ is large then ${ }^{p} \underline{n}_{x}^{\infty}={ }^{p} \underline{n}_{x}$.

\section{The new algorithm}

In this section we attempt to give the reader some idea of how we perform the calculations which led to our conjecture. The second author hopes to give more details in [26]. As explained in the previous section, the main theorems of $[9,21]$ (see also [1, 2]) imply that it is enough to calculate the $p$-canonical basis $\left\{{ }^{p} \underline{n}_{x} \mid x \in{ }^{f} W\right\}$ in the anti-spherical module $\mathrm{AS}_{v}$.

Let $\mathcal{H}$ denote the diagrammatic Hecke category associated to the affine Cartan matrix of type $\widetilde{A}_{n}$. Recall that $\mathcal{H}$ is built starting from a "realisation"

$$
\left(\left\{\alpha_{s}^{\vee}\right\}_{s \in S} \subset \mathfrak{h},\left\{\alpha_{s}\right\}_{s \in S} \subset \mathfrak{h}^{*}\right),
$$

where $\mathfrak{h}$ is a free and finite rank $\mathbb{Z}$-module, $\mathfrak{h}^{*}$ is its dual, and the usual formulas define a representation of $W$. It will be important below that our realisation is chosen so that the simple roots $\left\{\alpha_{s}\right\}_{s \in S} \subset \mathfrak{h}^{*}$ are linearly independent.

The Hecke category is defined over $\mathbb{Z}$. After fixing a prime $p$, extension of scalars yields the Hecke category $\mathcal{H}_{\mathbb{Z}_{p}}$ defined over the $p$-adic integers. Let $\mathcal{A S}_{\mathbb{Z}_{p}}$ denote the anti-spherical category over $\mathbb{Z}_{p}$ considered in $[15,21]$. It is a right $\mathcal{H}_{\mathbb{Z}_{p}}$-module category and one has a canonical identificiation of right $\left[\mathcal{H}_{\mathbb{Z}_{p}}\right]_{\oplus}=H$-modules:

$$
\mathrm{AS}_{v} \stackrel{\sim}{\rightarrow}\left[\mathcal{A S}_{\mathbb{Z}_{p}}\right]_{\oplus}
$$

\footnotetext{
${ }^{1}$ See [21, Conjecture 1.7] for a precise formulation. Note that this conjecture is expected to hold without any restrictions on $p$.

${ }^{2}$ This grading is conjectural in general. In $[1,2,4,9,21]$ its existence is established in many cases.
} 
where $[-]_{\oplus}$ denotes the split Grothendieck group of an additive category (see $[15,21]$ for more details). Under this isomorphism the classes of the indecomposable self-dual objects yields the $p$-canonical basis in $\mathrm{AS}_{v}$.

The anti-spherical category $\mathcal{A S}_{\mathbb{Z}_{p}}$ is defined by explicit generators and relations. Thus the question of determining the characters of its indecomposable objects (and hence the $p$-canonical basis) is a concrete question of finding idempotents in certain finite rank $\mathbb{Z}_{p}$-algebras. However these diagrammatic calculations are prohibitively difficult in all but the simplest cases.

Instead we exploit the philosophy of localisation. To $\mathcal{A S}_{\mathbb{Z}_{p}}$ are associated progressively simpler categories:

$$
\mathcal{A S}_{\mathbb{Z}_{p}} \rightsquigarrow \mathcal{A} \mathcal{S}_{\mathbb{Q}_{p}} \rightsquigarrow Q \otimes_{R} \mathcal{A} \mathcal{S}_{\mathbb{Q}_{p}},
$$

where $\rightsquigarrow$ denotes some form of localisation. The first localisation $\mathcal{A S} \mathcal{S}_{\mathbb{Q}_{p}}$ is obtained from $\mathcal{A S}_{\mathbb{Z}_{p}}$ by inverting $p$. The second is the main object of study of [15]: the polynomial ring $R=\mathbb{Q}_{p}[\widetilde{\alpha}]$ ( $\widetilde{\alpha}$ denotes the affine simple root) acts on the left on all hom spaces in $\mathcal{A} \mathcal{S}_{\mathbb{Q}_{p}}$, and after inverting $\widetilde{\alpha}$ one obtains $Q \otimes_{R} \mathcal{A S}_{\mathbb{Q}_{p}}$, where $Q$ denotes $\mathbb{Q}_{p}(\widetilde{\alpha})$. Somewhat surprisingly, this category is semi-simple [15]. (This is analogous to deformed category $\mathcal{O}$, which is semi-simple for generic parameters. A big difference in the current setting is that the deformation ring $R$ is always one dimensional.)

The algorithm we used to calculate the $p$-canonical basis now proceeds in two steps:

1. Firstly, $\mathcal{A S}_{\mathbb{Q}_{p}}$ is described as a quiver with relations, and the action of the Hecke category $\mathcal{H}_{\mathbb{Z}_{p}}$ on $\mathcal{A S}_{\mathbb{Q}_{p}}$ is described explicitly, in terms of the quiver. This is already a very non-trivial task, and is only feasible for $\mathrm{SL}_{2}, \mathrm{SL}_{3}$ and perhaps $\mathrm{SL}_{4}$. It is possible in these cases thanks to the localisation $Q \otimes_{R} \mathcal{A} \mathcal{S}_{\mathbb{Q}_{p}}$ (where any calculation can be reduced to a calculation in matrices with entries in $Q$ ), the fact that the Kazhdan-Lusztig conjectures hold in $\mathcal{A}_{\mathbb{Q}_{p}}$ (thus one has graded dimensions for hom spaces and knows how the generators of $\mathcal{H}_{\mathbb{Z}_{p}}$ act on the Grothendieck group etc.), and the fact that the Kazhdan-Lusztig theory of these anti-spherical modules in low rank is not too complicated (Kazhdan-Lusztig polynomials can be written down explicitly).

2. Secondly, via the above localisations one can describe $\mathcal{A} \mathcal{S}_{\mathbb{Z}_{p}}$ as a $\mathbb{Z}_{p}$-lattice inside $\mathcal{A S}_{\mathbb{Q}_{p}}$. Moreover, this lattice is the smallest lattice which contains the generating object and is stable under the action of the Hecke category $\mathcal{H}_{\mathbb{Z}_{p}}$. One may describe this lattice explicitly and inductively via the $\mathcal{H}_{\mathbb{Z}_{p}}$-action, using the philosophy of the light leaf basis. Details of how this is done in this setting will be contained in [26].

Remark 3.1. Recently, L.T. Jensen has done calculations which omit the first localisation and calculate the $p$-canonical basis using only the localisation $Q \otimes_{R} \mathcal{A} \mathcal{S}_{\mathbb{Q}_{p}}$. This is a much simpler approach, and appears (much to the second author's surprise!) not to be any slower than the approach described above. It may well be that Jensen's modification of the algorithm ends up being the more effective.

\section{Billiards}

For the rest of the paper we fix $G=\mathrm{SL}_{3}, \mathscr{X}=\mathbb{Z} \varpi_{1} \oplus \mathbb{Z} \varpi_{2}, \Sigma=\left\{\alpha_{1}, \alpha_{2}\right\}$ etc.

Fix $\ell \geq 1$. (For applications to representation theory $\ell$ will be prime, however for the moment $\ell$ can be any positive integer. Soon we will assume $\ell \geq 3$.) Elements of the set

$$
\mathcal{M}:=\mathbb{Z}_{\geq 0} \times\left\{v^{k} \mid k \in \mathbb{Z}\right\}
$$

will be called labels. Labels will often be denoted by an integer followed by a bracketed power of $v$; e.g., $51\left(v^{7}\right)$. 
A labelled point is an element of $\mathscr{X} \times \mathcal{M}$. Labelled points will usually be denoted $(\mu, m)$ with $\mu \in \mathscr{X}$ and $m \in \mathcal{M}$ or $\left(\mu, n, v^{k}\right)$ with $\mu \in \mathscr{X}$ and $n \in \mathbb{Z}_{\geq 0}$.

Our goal in this section is to describe an algorithm which produces a multiset (i.e., set with multiplicities) of labelled points via an inductive procedure. Throughout this section we work entirely with multisets. All operations (union, difference, ...) are to be understood in the context of multisets.

\subsection{Overview of the algorithm}

We view the dominant weights $\mathscr{X}_{+}$as the vertices of a directed graph $\Gamma$ with edges $\lambda \rightarrow \lambda+\gamma$ if $\lambda, \lambda+\gamma \in \mathscr{X}_{+}$and $\gamma \in\left\{\varpi_{1}, \varpi_{2}-\varpi_{1},-\varpi_{2}\right\}$ :

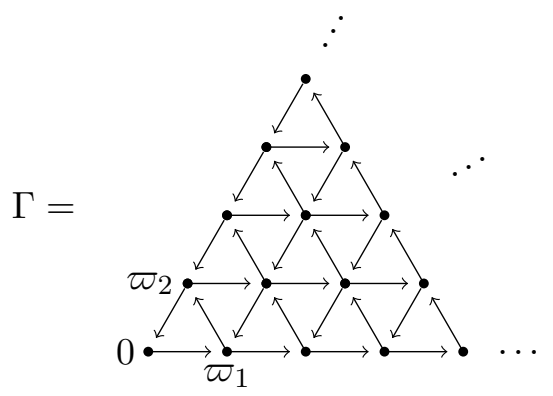

Our algorithm consists of three steps. We begin with the labelled point $\left(0,0, v^{0}\right)$ (our initial "seed"). Each step in our algorithm enlarges our multiset in a new direction, starting with seeds generated in the previous step. The first step extends our set along a wall of the dominant chamber in the direction of $\varpi_{1}$ to produce a set $X$. The second step extends our set along the walls of the $\ell$-alcoves to produce a multiset $Y$. Finally, the third step extends our multiset within the interior of each $\ell$-alcove to produce a multiset $Z$. In the third step, certain labelled points arising in the second step (seeds) gives rise to two spirals inside the interior of two adjacent alcoves which move like a billiard. Finally we consider

$$
\widetilde{Z}:=Z \backslash X,
$$

which is the object of our conjecture.

\subsection{Step 1: the wall of the dominant chamber}

Consider the full subgraph of $\mathscr{X}_{+}$consisting of multiples of $\varpi_{1}$ :

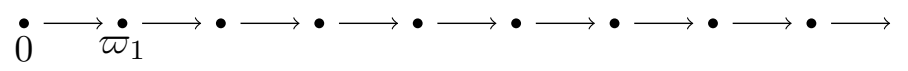

Consider a labelled point $m=\left(\mu, m\left(v^{k}\right)\right)$ such that $\mu$ belongs to this full subgraph. A small step produces the labelled point $\left(\mu^{\prime},(m+2)\left(v^{k}\right)\right)$ where $\mu \rightarrow \mu^{\prime}$ is the unique edge with source $\mu$. Consider the set $X$ obtained by repeatedly taking small steps beginning with the labelled point $\left(0,0\left(v^{0}\right)\right)$. In other words, $X$ is the set

$$
X=\left\{\left(k \omega_{1}, 2 k\left(v^{0}\right)\right) \mid k \in \mathbb{Z}_{\geq 0}\right\} .
$$

We designate the labelled points of the form $\left(\ell k, 2 k \ell\left(v^{0}\right)\right)$ with $k>0$ as seeds.

Remark 4.1. Of course we could have defined $X$ via (4.1) directly. We prefer the inductive definition, as it is closer in spirit to the more complicated definitions which will occur in the next two steps. 


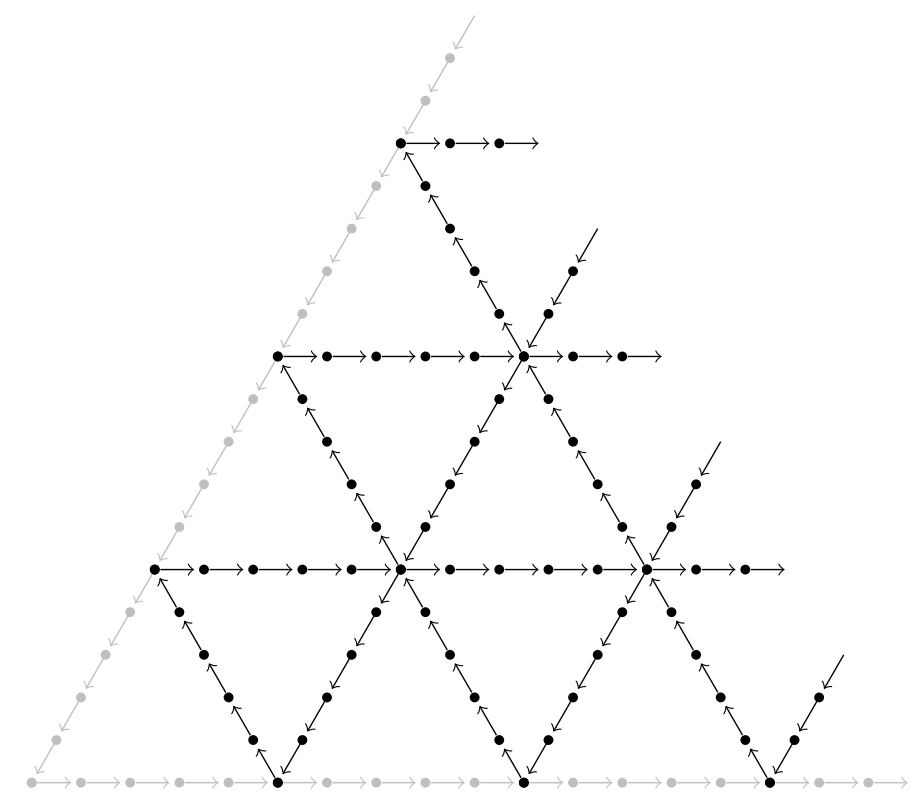

Figure 2. The directed graph $\Gamma_{\text {wall }}$.

\subsection{Step 2: dynamics on the walls}

From now on assume that $\ell \geq 3$. Consider the subgraph of $\Gamma$ with vertices $\lambda \in \mathscr{X}_{+}$such that $\left\langle\lambda, \alpha^{\vee}\right\rangle \in \ell \mathbb{Z}$ for some $\alpha \in \Phi_{+}$and edges $\left(\lambda, \lambda^{\prime}\right)$ such that $\left\langle\lambda, \alpha^{\vee}\right\rangle=\left\langle\lambda^{\prime}, \alpha^{\vee}\right\rangle \in \ell \mathbb{Z}$ for some $\alpha \in \Phi_{+}$. Let $\Gamma_{\text {wall }}$ denote the graph obtained by removing the gray edges and vertices as in Fig. 2. Points in $\mathscr{X}_{+}$or $\Gamma_{\text {wall }}$ such that $\left\langle\lambda, \alpha^{\vee}\right\rangle \in \ell \mathbb{Z}$ for all $\alpha \in \Phi_{+}$are called corner points. A point $\mu$ in $\mathscr{X}_{+}$of $\Gamma_{\text {wall }}$ is an almost corner if there exists a corner point $c$ and an arrow $c \rightarrow \mu$ in $\mathscr{X}_{+}$.

Fix a labelled point $\left(\mu, n\left(v^{k}\right)\right)$ with $\mu \in \Gamma_{\text {wall }}$. We assume that $\mu$ is such that there is a unique edge with source $\mu$. From $\left(\mu, n\left(v^{k}\right)\right)$ one may obtain new labelled points as follows:

1. A rest produces the labelled point $\left(\mu,(n+3)\left(v^{k+1}\right)\right)$.

2. A small step produces (as above) the labelled point $\left(\mu^{\prime},(n+2)\left(v^{k}\right)\right)$, where $\mu \rightarrow \mu^{\prime}$ is the unique edge in $\Gamma_{\text {wall }}$ with source $\mu$.

3. A giant leap produces either one or two new labelled points, and is only possible if $\mu$ is not a corner or almost corner point. Define $d$ to be the direction of the unique arrow with source $\mu$. First we proceed $j<\ell-1$ steps in the graph $\Gamma_{\text {wall }}$ in direction $d$ until we reach a corner point. We then proceed for another $\ell-1-j$ steps in all directions from the corner point which do not agree with the direction $d$. (There are either one or two such directions.) A giant leap consists of the resulting points, which are labelled by $(n+2 \ell+1)\left(v^{k+1}\right)$ (see Fig. 3).

Now suppose that we are given a labelled point $q=\left(\mu, n, v^{k}\right)$ as above (i.e., such that there is a unique edge in $\Gamma_{\text {wall }}$ with source $\mu$ ). We now describe a way of producing a multiset of labelled points (all with multiplicity 1 ) beginning with $q$, some of which are designated as seeds:

1. If $\mu$ is a corner point. We produce $\ell$ new points as follows: we take $\ell-1$ small steps to produce labelled points $m=m_{1}, \ldots, m_{\ell-1}$ and then rest to produce a new labelled point $m_{\ell}$. The final labelled point $m_{\ell}$ is designated as a seed. (See Fig. 4.)

2. If $\mu$ is an almost corner point. We produce $\ell$ new points as follows: let $m=m_{1}$ and rest once to produce a new labelled point $m_{2}$, now take $\ell-2$ small steps starting with $m_{2}$ to 

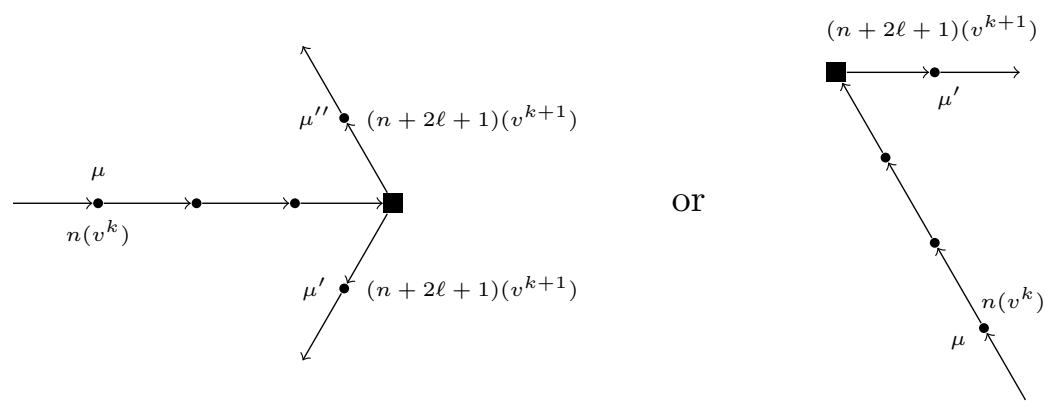

Figure 3. Performing a giant leap with $\ell=5$. (Squares denote corner points.)
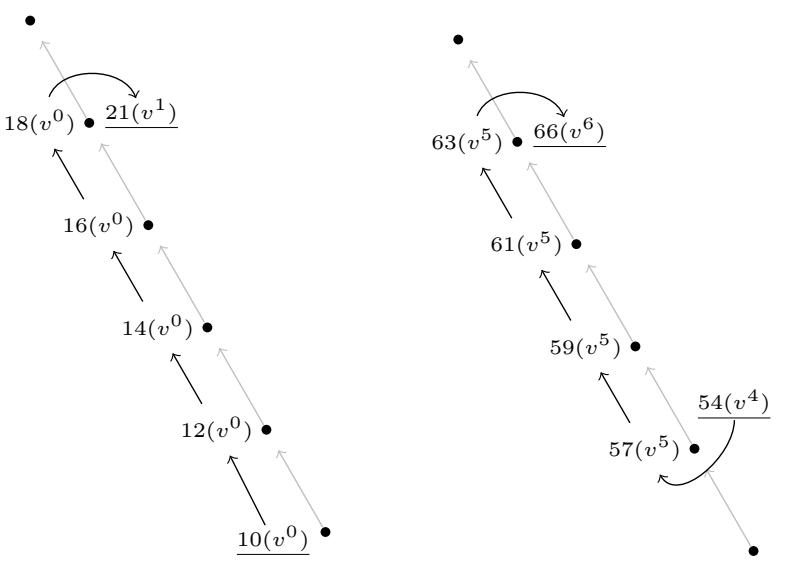

Figure 4. An illustration of cases (1) and (2) when $\ell=5$. Seeds are underlined.

produce $\ell-2$ labelled points $m_{3}, \ldots, m_{\ell-1}$ and finally rest once more to produce a final labelled point $m_{\ell}$. The final labelled point $m_{\ell}$ is designated as a seed. (See Fig. 4.)

3. If $\mu$ is neither a corner nor an almost corner point. We take a giant leap to produce one or two new labelled points, each of which are designated seeds.

We now iterate this process as follows. Starting with $q$ we produce a sequence of multisets $Q_{1}, Q_{2}, Q_{3}, \ldots$, where $Q_{1}$ (resp. $Q_{i}$ for $i>1$ ) is obtained by applying the above procedure to $q$ (resp. to each seed in $Q_{i-1}$ ). We say that the union (as multisets)

$$
Q=\bigcup_{i \geq 0} Q_{i}
$$

is the result of applying dynamics on the walls to the seed $q$.

Example 4.2. Fig. 5 gives an example with $\ell=5$. Our initial seed is the unique point with label $10\left(v^{0}\right)$. We illustrate a few iterations of the above algorithm. Seeds are underlined. Note that one more iteration of the algorithm will produce the labelled point $\left(3 \varpi_{1}+7 \varpi_{2}, 88\left(v^{8}\right)\right)$ with multiplicity 2.

Now consider our set $X$ from the previous step. We apply dynamicson walls to each seed in $X$ (i.e., each labelled point of the form $\left(k \ell \varpi_{1}, 2 k \ell\left(v^{0}\right)\right)$ with $\left.k>0\right)$ to generate a multiset $Y_{k}$. We now define a new multiset

$$
Y:=X \cup \bigcup_{k>0} Y_{k}
$$

We also remember (for the purposes of the next step) which elements of $Y$ were designated seeds. 


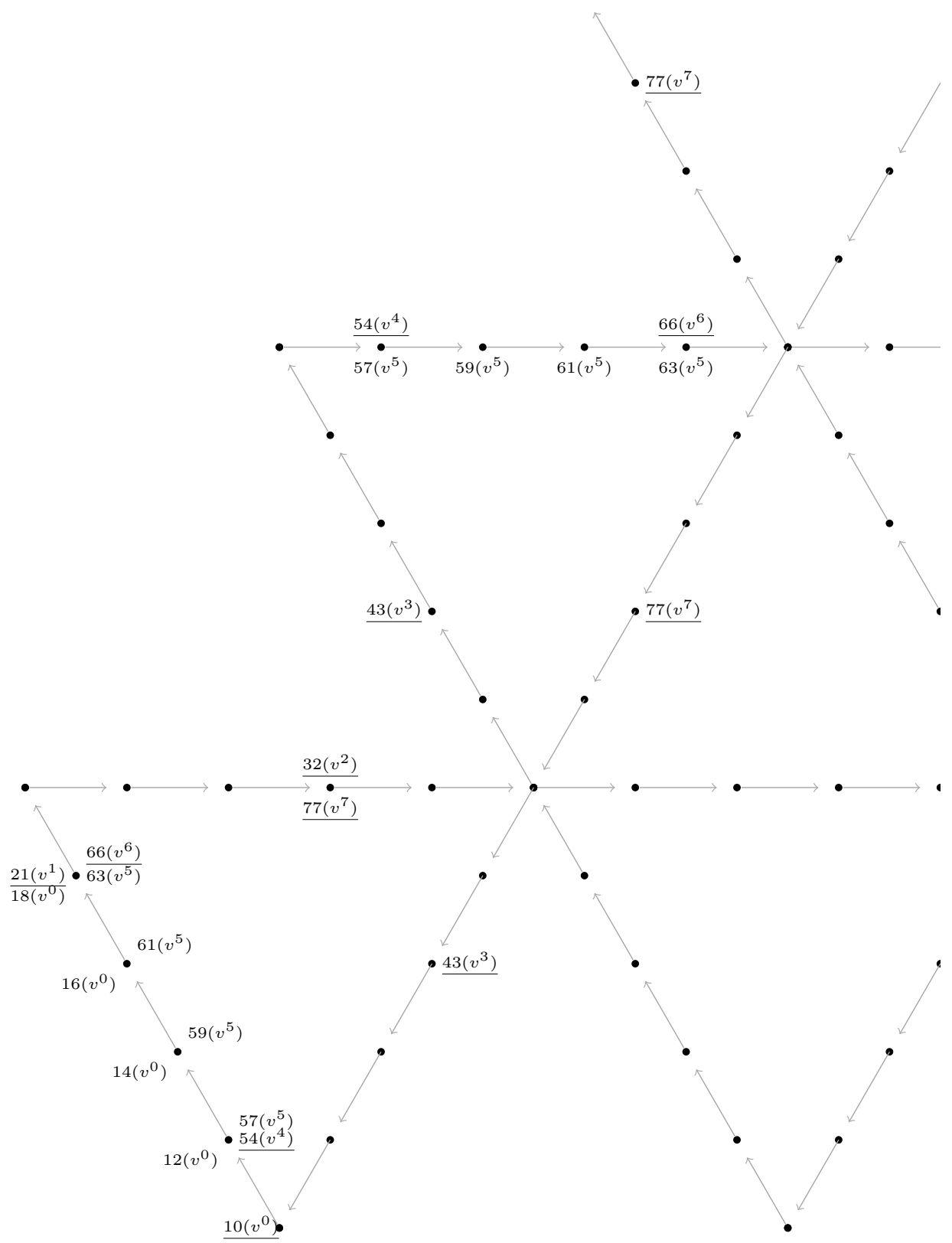

Figure 5. Dynamics on the walls with $\ell=5$.

Remark 4.3. Consider dynamics on the wall restricted to the full subgraph displayed in Fig. 6 with seed $q=\left(\mu, n\left(v^{k}\right)\right)$. Then 4 iterations of the above algorithm yields the following points and labels (see Fig. 6):

$$
\begin{array}{lcl}
u, u^{\prime} & \text { with label } \quad(n+11)\left(v^{k+1}\right), & \\
u_{i}, u_{i}^{\prime} \quad \text { with label } \quad(n+14+2(i-1))\left(v^{k+2}\right) & \text { for } i=1,2,3,4, \\
v, v^{\prime} \quad \text { with label } \quad(n+23)\left(v^{k+3}\right), & \\
w, w^{\prime} \quad \text { with label } \quad(n+34)\left(v^{k+4}\right), &
\end{array}
$$

and then $\mu$ with label $(n+45)\left(v^{k+5}\right)$ and multiplicity 2. (The point is that there are two directed paths leading from $\mu$ back to itself, which causes the multiplicity to double.) Repeating this algorithm $4 i$ times leads to $q_{i}:=\left(\mu, n+45 i\left(v^{k+5 i}\right)\right)$ with multiplicity $2^{i}$. From this observation 


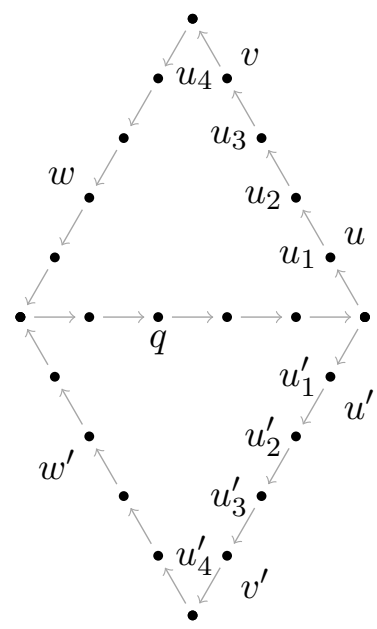

Figure 6. Subgraph demonstrating exponential growth of multiplicities for $\ell=5$.

one deduces easily that for $\ell=5$ the set $Y$ contains labelled points whose multiplicity grows exponentially in $n$. Similar considerations show that the same statement about $Y$ is true for any $\ell$.

\subsection{Step 3: billiards in an alcove}

We now describe an algorithm which produces for each seed in $Y$ a multiset of labelled points. We need a little more notation. We consider the dominant weights $\mathscr{X}_{+}$as embedded in the vector space

$$
\mathscr{X}_{\mathbb{R}}:=\mathscr{X} \otimes_{\mathbb{Z}} \mathbb{R}
$$

which we regard as a Euclidean space for some $W_{f}$-invariant bilinear form. An $\ell$-alcove is a connected component of the complement

$$
\mathscr{X}_{\mathbb{R}} \backslash \bigcup_{\alpha \in \Phi_{+}^{\vee}}\left\{\lambda \mid\left\langle\alpha^{\vee}, \lambda\right\rangle \in \ell \mathbb{Z}\right\} .
$$

An $\ell$-regular point is a point which belongs to some $\ell$-alcove. An $\ell$-alcove is dominant if it is contained in the real cone generated by $\mathscr{X}_{+}$.

Now consider a seed $q=(\mu, m) \in Y$. We want to associate a multiset of labelled points $\Lambda_{q}$ to $q$. If $\mu$ is a corner or almost corner, we set $\Lambda_{q}:=\varnothing$.

From now on we assume that $q$ is neither a corner or almost corner. In this case $\mu$ belongs to the closure of two dominant $\ell$-alcoves, $A^{\prime}$ and $A^{\prime \prime}$. We perform an identical procedure for both alcoves, so fix one such alcove and call it $A$. Let $\Delta$ denote the full subgraph of $\Gamma$ consisting of points belonging to $\bar{A}$. Then $\Delta$ has one of the following forms:

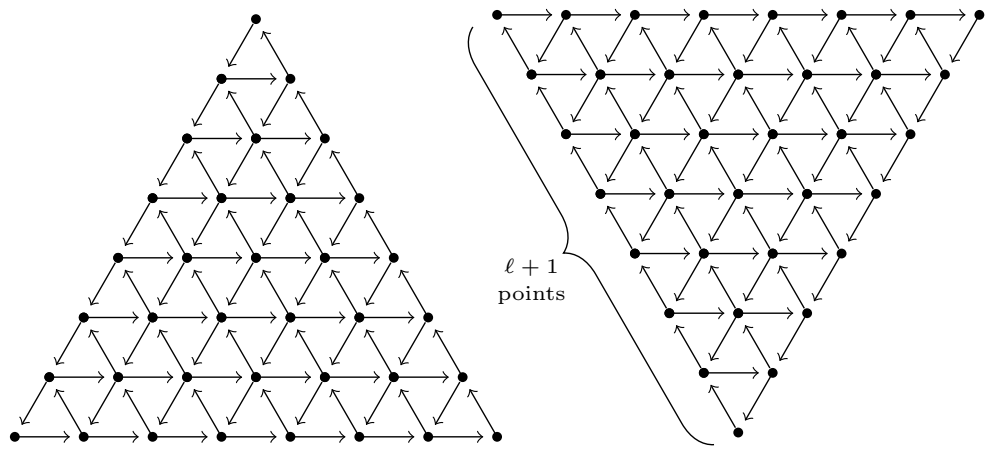


Points of $\Delta$ belong to $\bar{A}$ but not to $A$ are called wall points. Note that our starting point $\mu$ is a wall point.

Variables and their initialisations: Let $\left(\mu_{\text {start }}, m_{\text {start }}\right):=(\mu, m)$ and let $d_{\text {start }}$ denote the unique edge of $\Delta$ with source $\mu$ and target an interior point of $\Delta$. To begin with, set $\Lambda:=$ $\left\{\left(\mu_{\text {start }}, m_{\text {start }}\right)\right\}$. In the algorithm we need the following variables, which are initialised as follows:

$$
\begin{array}{ll}
\text { CurrentPoint }:=\mu_{\text {start }}, & \text { CurrentLabel }:=m_{\text {start }}, \\
\text { CurrentDirection }:=d_{\text {start }}, & \text { CurrentSign }:=1 .
\end{array}
$$

The loop: The multiset of labelled points is obtained by repeating the following ad infinitum. Let $\mu_{\text {new }}$ denote the point obtained by moving from CurrentPoint one step in CurrentDirection. Write CurrentLabel as $n\left(v^{k}\right)$. Two possibilities may occur:

1. $\mu_{\text {new }}$ is $\ell$-regular:

$$
\begin{aligned}
& \text { CurrentLabel }:=(n+2)\left(v^{k}\right), \quad \text { CurrentPoint }:=\mu_{\text {new }}, \\
& \text { add (CurrentPoint, CurrentLabel) to } \Lambda .
\end{aligned}
$$

(The variables CurrentDirection and CurrentSign remain unchanged.)

2. CurrentPoint is $\ell$-regular and $\mu_{\text {new }}$ is a wall point:

$$
\begin{array}{ll}
\text { CurrentLabel }:=(n+3)\left(v^{k+\text { CurrentSign }}\right), & \text { CurrentDirection }:=d_{\text {new }}, \\
\text { CurrentSign }:=- \text { CurrentSign, } & \text { add (CurrentPoint, CurrentLabel) to } \Lambda .
\end{array}
$$

Here $d_{\text {new }}$ denotes the vector obtained by reflecting CurrentDirection in the wall on which $\mu_{\text {new }}$ lies. (The variable CurrentPoint remains unchanged.)

Example 4.4. We illustrate the first few steps of the algorithm in some examples. In each case the starting point $\mu_{\text {start }}$ and its label are underlined. The successive values of CurrentLabel and CurrentPoint are obtained by following the arrows.

1. A reasonably generic example with $\ell=11$ :

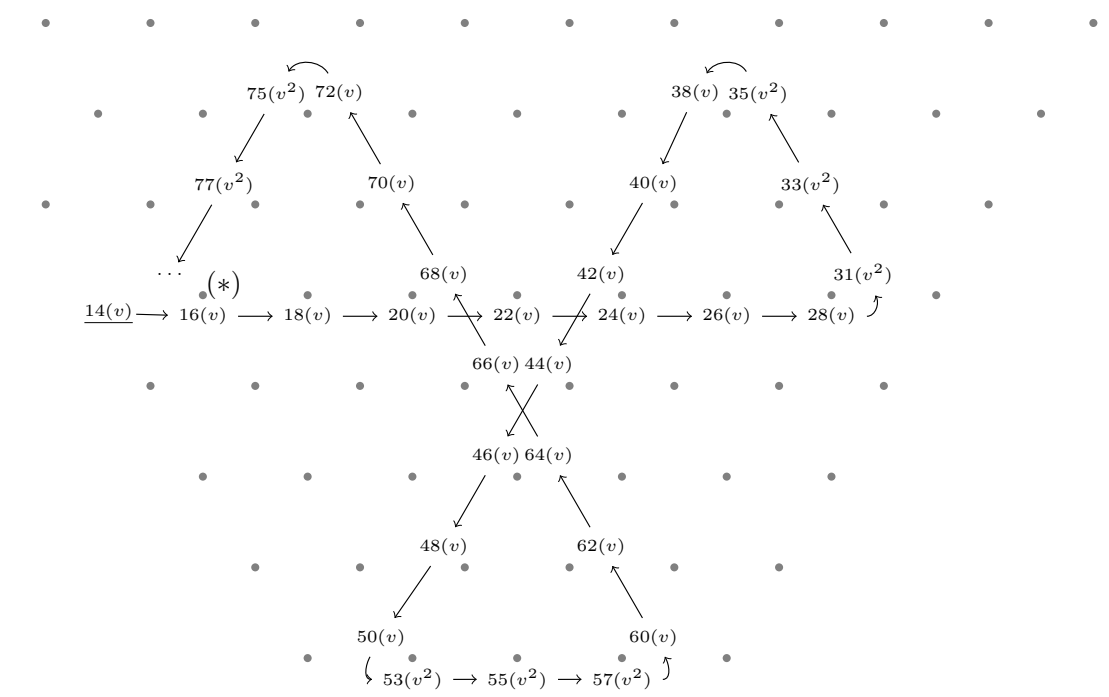


If we focus on the point marked with an asterix $\left(^{*}\right)$ we obtain the labels:

$$
\left\{16(v), 79\left(v^{2}\right), 82(v), 145\left(v^{2}\right), 148(v), 211\left(v^{2}\right), 214(v), 277\left(v^{2}\right), 280(v), \ldots\right\} .
$$

2. An interesting example with $\ell=5$ (we display both alcoves):

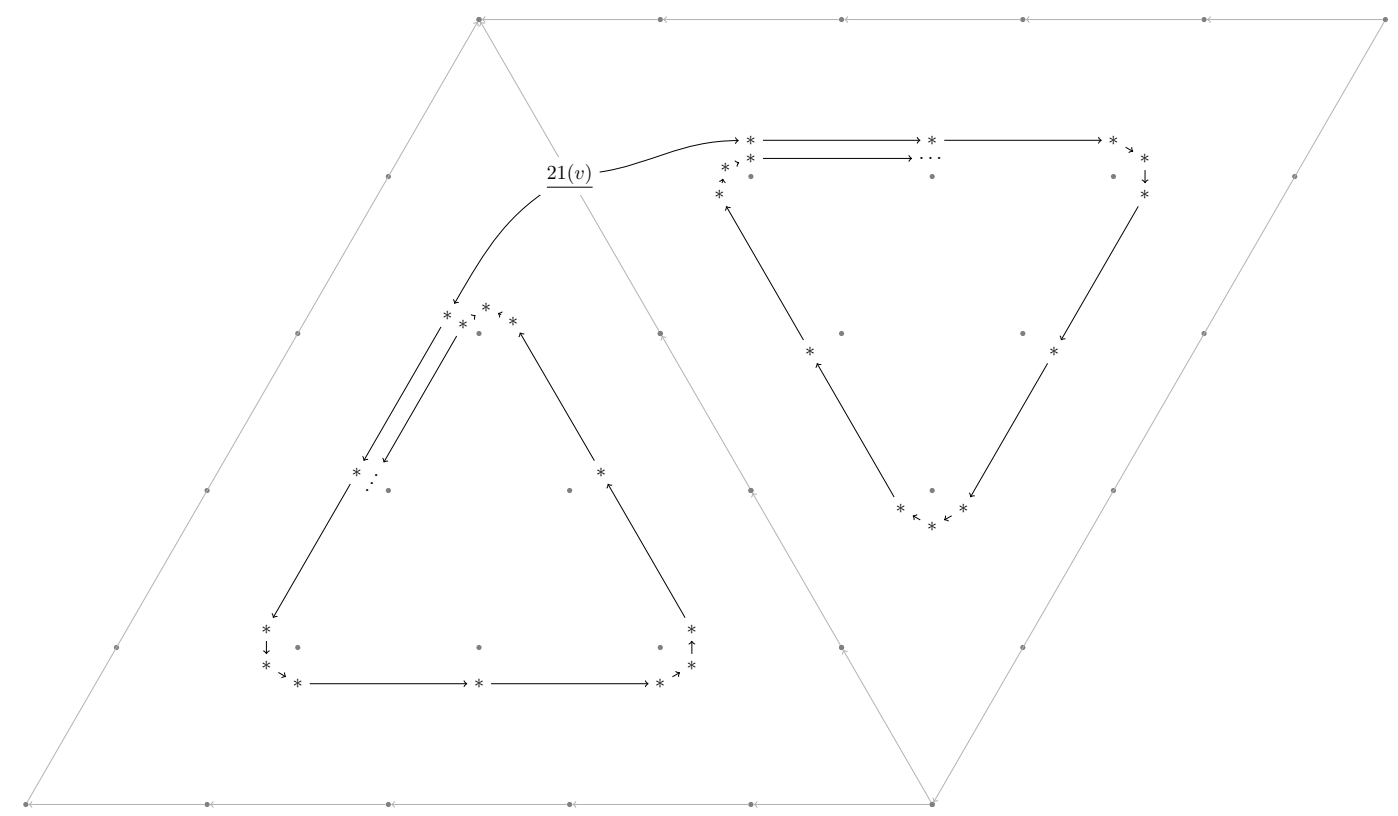

In either alcove the sequence of labels obtained by following the arrows beginning at $21(v)$ is as follows:

$$
\begin{gathered}
23(v), 25(v), 27(v), 30 \mathfrak{i}\left(v^{2}\right), 33(v), 35(v), 37(v), 40\left(v^{2}\right), \\
43(v), 45(v), 47(v), 50\left(v^{2}\right), 53(v), \ldots
\end{gathered}
$$

3. Another example with $\ell=5$ (again we display both alcoves):

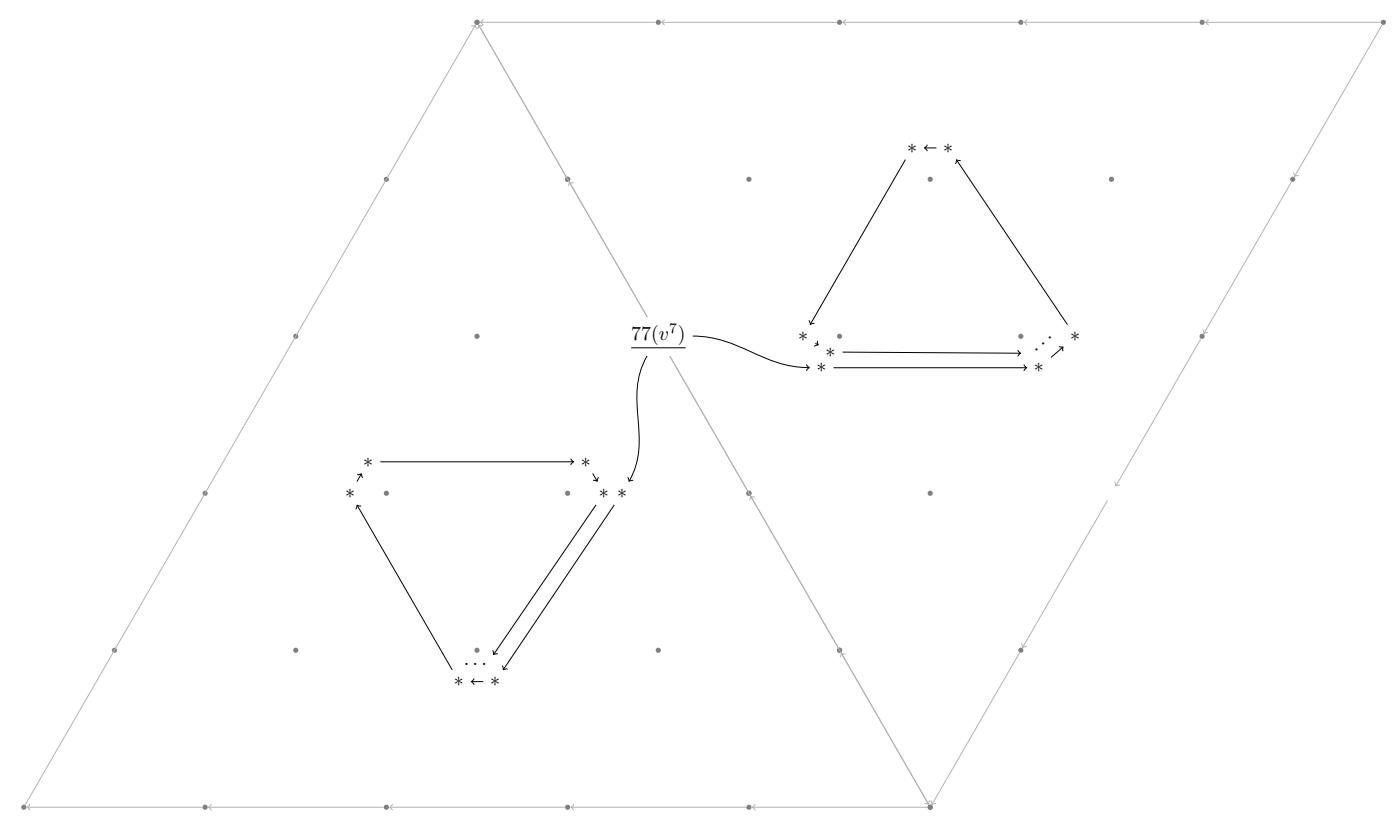

In either alcove the sequence beginning at $77\left(v^{7}\right)$ is

$$
79\left(v^{7}\right), 81\left(v^{7}\right), 84\left(v^{8}\right), 86\left(v^{8}\right), 89\left(v^{7}\right), 91\left(v^{7}\right), \ldots
$$


4. Finally, an example with $\ell=3$ (again we display both alcoves):

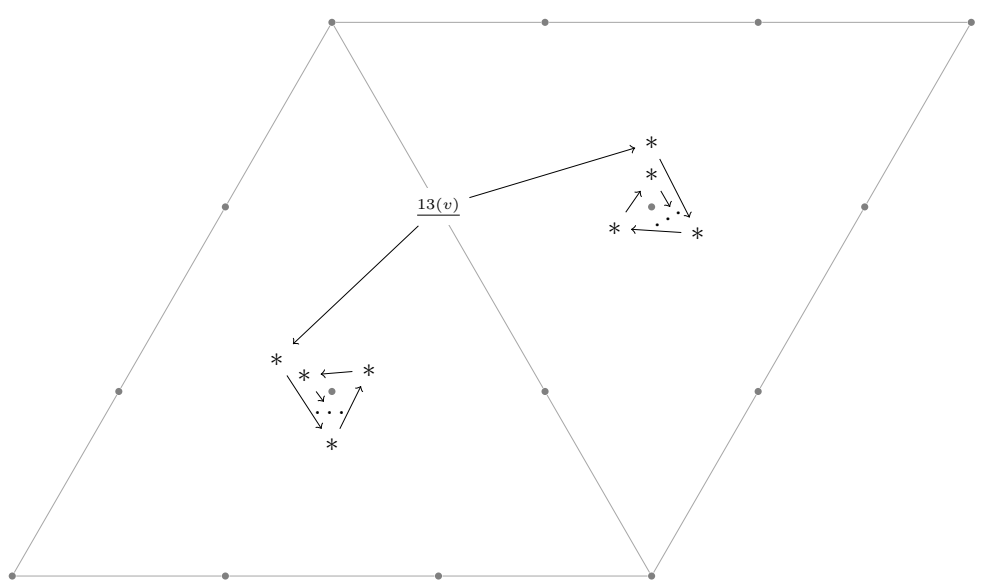

The sequence at either middle vertex is: $15(v), 18\left(v^{2}\right), 21(v), 24\left(v^{2}\right), 27(v), \ldots$

Now, with $q=(\mu, m)$ as above (so that $\mu$ is neither a corner or almost corner) we apply the above algorithm to both alcoves which contain $\mu$ in their closure to produce multisets $\Lambda^{\prime}$ and $\Lambda^{\prime \prime}$. We then remove $q$ from both, and define $\Lambda_{q}$ to be the union of the resulting multisets. (Thus $\Lambda_{q}$ contains infinitely many points from both alcoves, but does not contain $q$ itself.)

The above algorithm produces, for each each seed $q \in Y$, a multiset $\Lambda_{q}$. We define $Z$ to be the union

$$
Z:=Y \cup \bigcup_{\text {seeds }} \bigcup_{q \in Y} \Lambda_{q}
$$

Finally, we define $\widetilde{Z}:=Z \backslash X$.

Example 4.5. In Fig. 1 we display $\widetilde{Z}$ for $\ell=5$ and all labelled points $\left(\mu, n\left(v^{k}\right)\right)$ with $n \leq 82$. In the notation of Section 6 , the labelled points corresponding to $\mu \in \mathscr{X}_{+}$are displayed in the smaller of the two alcoves contained in $B_{\mu}$. The reader is referred to [25] for further examples.

Remark 4.6. For all $\left(\mu, n\left(v^{k}\right)\right) \in \widetilde{Z}$ it is easy to see that $\mu \in \mathscr{X}_{++}:=\mathbb{Z}_{>0} \varpi_{1} \oplus \mathbb{Z}_{>0} \varpi_{2}$.

\section{An altenative construction}

In this section we outline an alternative construction of the multiset $\widetilde{Z}$, which is more natural in some respects.

In the previous section we regarded the dominant weights $\mathscr{X}_{+}$as the vertices of a directed graph. An important difference in the current construction is that now we consider all weights $\mathscr{X}$. That is, we consider the elements of $\mathscr{X}$ as the vertices of a directed graph with edges $\lambda \rightarrow \lambda+\gamma$ if $\lambda, \lambda+\gamma \in \mathscr{X}_{+}$and $\gamma \in\left\{\varpi_{1}, \varpi_{2}-\varpi_{1},-\varpi_{2}\right\}$. We write $\mu \rightarrow \mu^{\prime}$ to indicate that there is a directed edge from $\mu$ to $\mu^{\prime}$.

We use similar terminology to earlier: the notions of a wall point, corner point, almost corner and $\ell$-regular point extend in an obvious way to $\mathscr{X}$. Note that almost corners are necessarily wall points.

\section{$5.1 \quad$ Trees}

For us a rooted tree is what many call an "arborescence": a directed graph with a distinguished vertex (the source) such that there is a unique directed path from the source to any other vertex. ${ }^{3}$

\footnotetext{
${ }^{3}$ This is equivalent in an obvious way to the usual notion of rooted tree.
} 
Given two rooted trees $A$ and $B$, denote by $A * B$ the rooted tree obtained by adjoining one copy of $B$ to each sink in $A$ at the source of $B$. That is, if $t_{1}, \ldots, t_{k}$ denote the sinks of $A$ and we denote by $b_{1}, \ldots, b_{k}$ the sources in $B \sqcup B \sqcup \cdots \sqcup B$ ( $k$ factors), then

$$
A * B:=(A \sqcup B \sqcup B \sqcup \cdots \sqcup B) /\left(t_{i} \sim b_{i}\right) .
$$

This operation is associative. The image of $A$ (resp. a copy of $B$ ) under this quotient map will be called a component of type $A$ (resp. $B$ ).

Recall that $\ell \geq 3$ is fixed. Consider the two rooted trees:

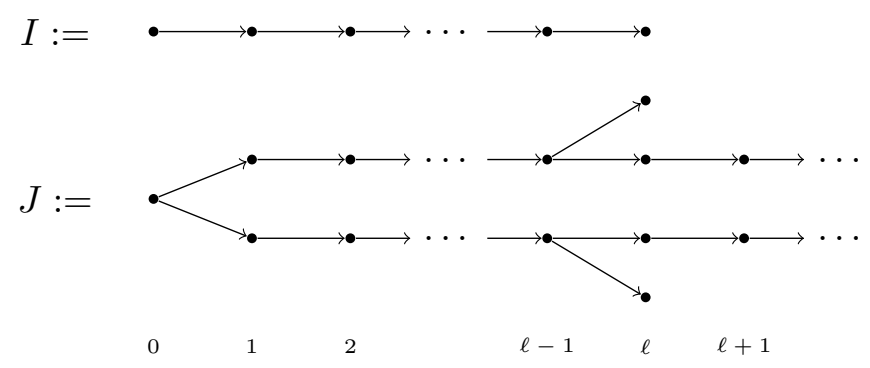

The integers indicate the distance from the unique source in each graph.

Example 5.1. We illustrate the operation $*$ with our graphs $I$ and $J$. For $\ell=3$, the graph $I * J * J$ looks as follows:

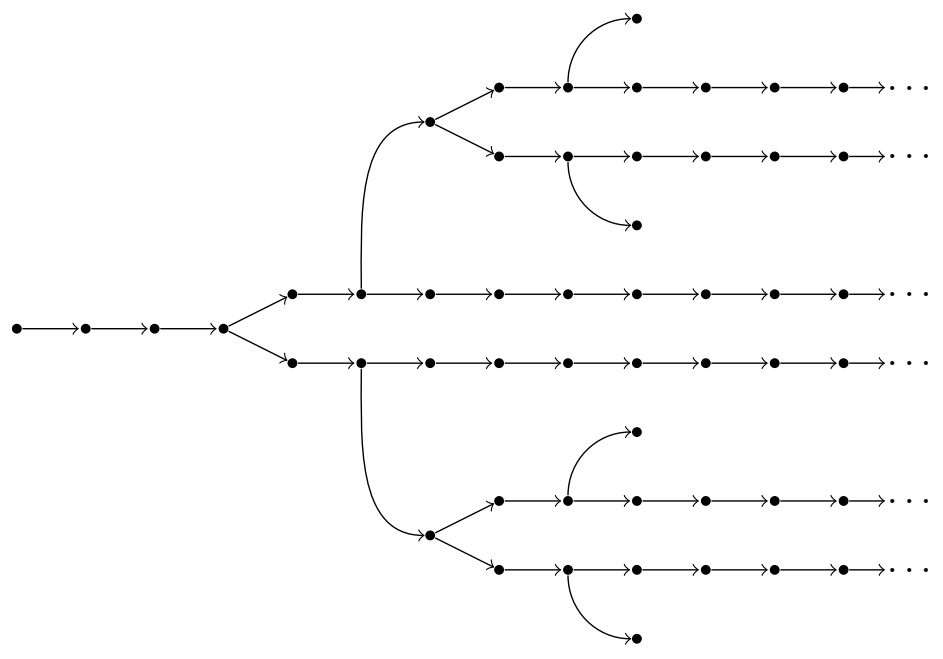

Now define:

$$
J_{\infty}:=\left(I * J^{* \ell-1}\right)^{* \infty}:=\left(I * J^{* \ell-1}\right) *\left(I * J^{* \ell-1}\right) *\left(I * J^{* \ell-1}\right) * \cdots
$$

Recall the notion of component from above. We say that $v \in J_{\infty}$ is of wall type if it lies in a component of type $I$ or is the source in a component of type $J$. We say that $v$ is of almost corner type if it is the source in a component of type $I$. (Thus if $v \in J_{\infty}$ is of almost corner type then it is also of wall type.)

Example 5.2. We continue Example 5.1 with $\ell=3$. The points of wall type (resp. of almost 
corner type) in $J_{\infty}$ are depicted as open (resp. filled) circles:

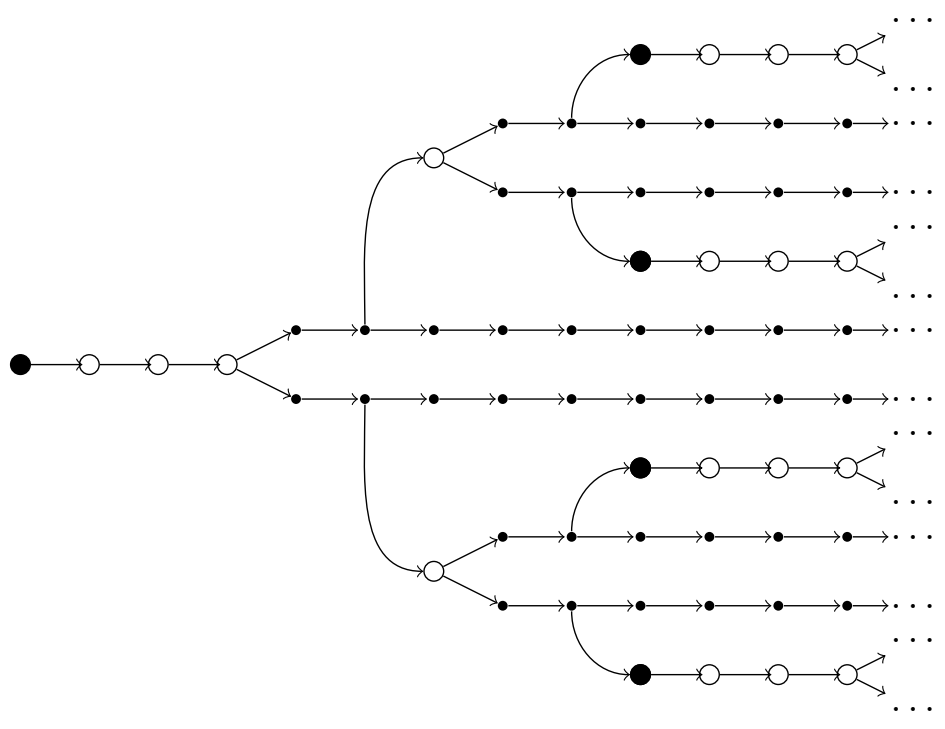

\subsection{The map}

Fix an almost corner $\lambda$. We will construct a map

$$
\Phi=\Phi_{\lambda}: J_{\infty} \rightarrow \mathscr{X}
$$

It will have the following two properties:

1. If $v \rightarrow v^{\prime}$ in $J_{\infty}$ then either $\Phi(v)=\Phi\left(v^{\prime}\right)$ or $\Phi(v) \rightarrow \Phi\left(v^{\prime}\right)$ in $\mathscr{X}$.

2. For $v \in J_{\infty}, \Phi(v)$ is a wall point (resp. an almost corner) if and only if $v$ is of wall type (resp. of almost corner type).

Because $J_{\infty}$ is the union of components of types $I$ or $J$ it will suffice to define the image of $\Phi$ on the source of $J_{\infty}$, and then define it on each component of type $I$ or $J$ inductively. This is what we do now:

Rule 0: Let $v_{0}$ denote the source of $J_{\infty}$. Set $\Phi\left(v_{0}\right)=\lambda$ (our fixed almost corner).

Rule 1: Let $I^{\prime} \subset J_{\infty}$ denote a component of type $I$ and let $v_{0}^{\prime}$ denote the source of $I^{\prime}$. Suppose that $\mu:=\Phi\left(v_{0}^{\prime}\right)$ is defined, but that $\Phi$ is not defined on the rest of $I^{\prime}$. We define $\Phi$ on $I^{\prime}$ as follows:

$$
\mu_{1} \rightarrow \mu_{1} \rightarrow \mu_{2} \rightarrow \ldots \rightarrow \mu_{\ell-2} \rightarrow \mu_{\ell-1} \rightarrow \mu_{\ell-1} .
$$

Here $\mu=\mu_{1}, \mu_{2}, \ldots, \mu_{\ell}$ are uniquely determined by the requirement that $\mu=\mu_{1}$ and $\mu_{1} \rightarrow \mu_{2} \rightarrow$ $\cdots \rightarrow \mu_{\ell-1}$ are all wall points. (The fact that $\mu_{1}, \ldots, \mu_{\ell-1}$ are well defined is a consequence of property (2) above: $\Phi\left(v_{0}^{\prime}\right)$ is an almost corner.)

Rule 2: Let $J^{\prime} \subset J_{\infty}$ denote a component of type $J$ and let $v_{0}^{\prime}$ denote the source of $J^{\prime}$. Suppose that $\mu:=\Phi\left(v_{0}^{\prime}\right)$ is defined, but that $\Phi$ is not defined on all of $J^{\prime}$.

By property (2) above $\mu$ is a wall-point but is not an almost corner. Hence there exist two edges $d^{\prime}, d^{\prime \prime}$ with source $\mu$ and image an $\ell$-regular point. For $d \in\left\{d^{\prime}, d^{\prime \prime}\right\}$, define a sequence $\left\{\left(\mu_{i}, d_{i}\right)\right\}_{i \geq 0}$ as follows:

1) $\left(\mu_{0}, d_{0}\right):=(\mu, d)$;

2) if $\mu_{i}+d_{i}$ is an interior point, define $\left(\mu_{i+1}, d_{i+1}\right):=\left(\mu_{i}+d_{i}, d_{i}\right)$; 
$3)$ if $\mu_{i}+d_{i}$ is a wall point, define $\left(\mu_{i+1}, d_{i+1}\right):=\left(\mu_{i}, r\left(d_{i}\right)\right)$, where $r$ denotes the reflection in the (unique) wall containing $\mu_{i}+d_{i}$.

Denote by $\left\{\left(\mu_{i}^{\prime}, d_{i}^{\prime}\right)\right\}_{i \geq 0}$ (resp. $\left.\left.\left\{\left(\mu_{i}^{\prime \prime}, d_{i}^{\prime \prime}\right)\right)\right\}_{i \geq 0}\right)$ the sequences associated to $d=d^{\prime}$ (resp. $\left.d=d^{\prime \prime}\right)$.

We define $\Phi$ on $J^{\prime}$ as follows:

$$
\mu_{0}^{\prime}=\mu_{0}^{\prime \prime} \longrightarrow \mu_{1}^{\prime} \longrightarrow \mu_{2}^{\prime} \longrightarrow \cdots \longrightarrow \mu_{\ell-1}^{\prime} \stackrel{\mu_{1}^{\prime \prime} \longrightarrow \mu_{\ell}^{\prime} \longrightarrow \mu_{\ell+1}^{\prime} \longrightarrow \cdots}{\longrightarrow} \longrightarrow \mu_{\ell-1}^{\prime \prime} \underset{\mu_{\ell-1}^{\prime \prime}+d_{\ell-1}^{\prime \prime} \longrightarrow \mu_{\ell+1}^{\prime \prime} \longrightarrow \cdots}{\longrightarrow} \longrightarrow \cdots
$$

(Note that $\mu_{\ell-1}^{\prime}+d_{\ell-1}^{\prime}$ and $\mu_{\ell-1}^{\prime \prime}+d_{\ell-1}^{\prime \prime}$ are wall points.)

In order for the above definition to be well defined the we should check that properties (1) and (2) are satisfied at each step. properties (1) and (2) for wall points are immediate from the definitions. Property (2) for almost corner points follows from the following observation: suppose that $J^{\prime} \subset J_{\infty}$ is a component of type $J$, let $v_{0}^{\prime}$ denote its source, and let $v_{1}^{\prime}, v_{1}^{\prime \prime}$ denote the two sinks (so that $v_{1}^{\prime}$ and $v_{1}^{\prime \prime}$ are of wall type in $J_{\infty}$ ). Then if $\Phi\left(v_{0}^{\prime}\right)$ is of distance $k \leq \ell-1$ from a corner point, then $\Phi\left(v_{1}^{\prime}\right)$ and $\Phi\left(v_{1}^{\prime \prime}\right)$ are of distance $k-1$ from a corner point.

Example 5.3. We illustrate $\Phi$ on a component of type $J$ with $\ell=5$ (the source is marked with a circle):

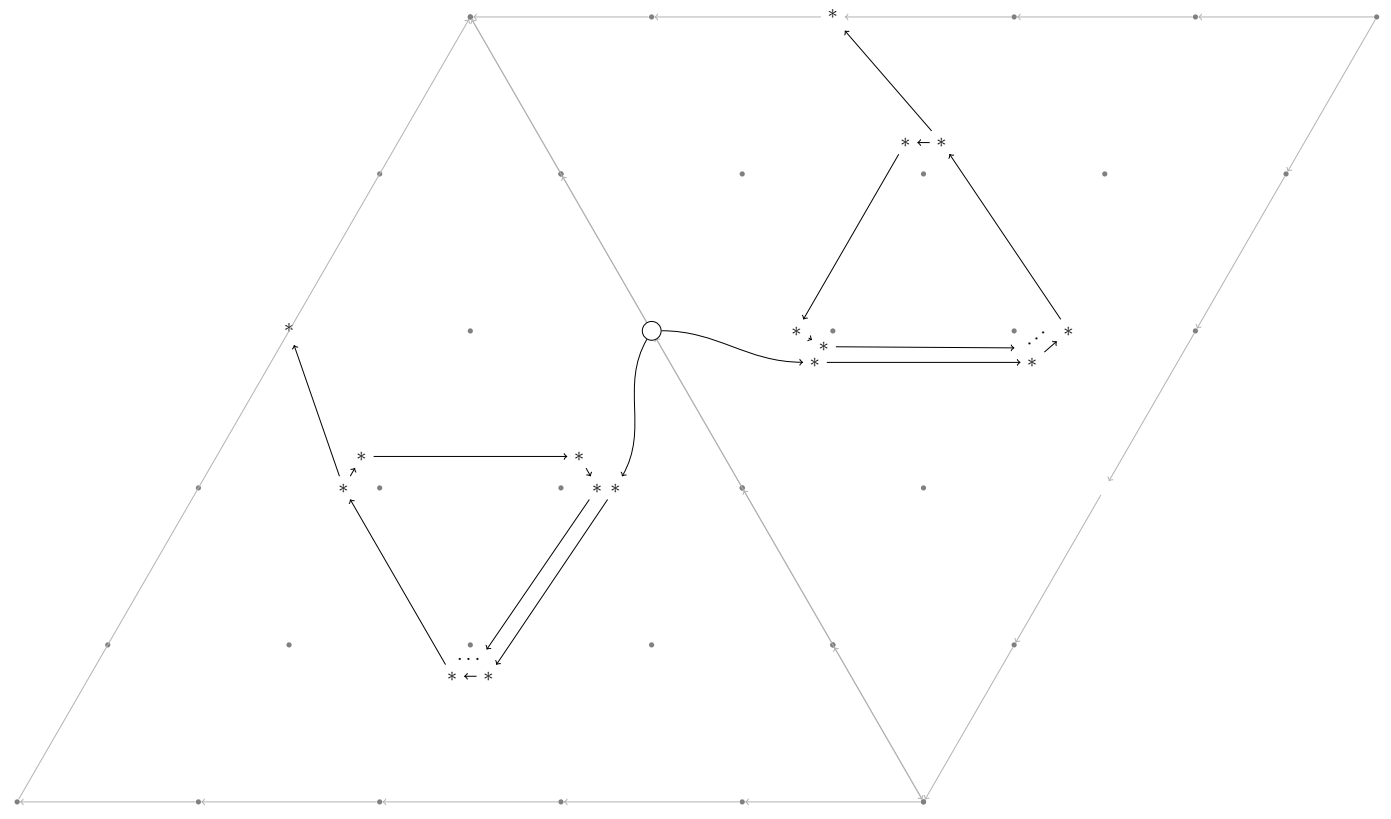

Note the similarity to the previous section. The only difference is the arrow joining an $\ell$-regular point to a wall point.

\subsection{Extending $\Phi$}

Let $v_{0}$ denote the source of $J_{\infty}$. For any choice of label $n\left(v^{k_{0}}\right)$, we inductively extend $\Phi$ to produce a map

$$
\widetilde{\Phi}=\widetilde{\Phi}_{\lambda, n\left(v^{k_{0}}\right)}: J_{\infty} \rightarrow \mathscr{X} \times \mathcal{M}
$$


such that $\widetilde{\Phi}\left(v_{0}\right)=\left(\lambda, n\left(v^{k_{0}}\right)\right)$. We proceed as follows: Suppose that $v \rightarrow v^{\prime}$ in $J_{\infty}$ and that $\widetilde{\Phi}$ is defined on $v$ but not on $v^{\prime}$, and let $\widetilde{\Phi}(v)=\left(\Phi(v), m\left(v^{k}\right)\right)$. Then $\widetilde{\Phi}\left(v^{\prime}\right)=\left(\Phi\left(v^{\prime}\right), m^{\prime}\left(v^{k^{\prime}}\right)\right)$ with

$$
m^{\prime}:= \begin{cases}m+2 & \text { if } \Phi(v) \neq \Phi\left(v^{\prime}\right) \\ m+3 & \text { if } \Phi(v)=\Phi\left(v^{\prime}\right)\end{cases}
$$

and

$$
k^{\prime}:= \begin{cases}k & \text { if } \Phi(v) \neq \Phi\left(v^{\prime}\right) \\ k+1 & \text { if } \Phi(v)=\Phi\left(v^{\prime}\right) \text { and } \\ & v \rightarrow v^{\prime} \text { belongs to a component of type } I \\ k \pm 1 & \text { if } \Phi(v)=\Phi\left(v^{\prime}\right) \text { and } \\ & v \rightarrow v^{\prime} \text { belongs to a component of type } J .\end{cases}
$$

The sign ambiguity in the final case is resolved as follows: suppose that $J^{\prime} \subset J_{\infty}$ is a component of type $J, v_{0}^{\prime}$ is its initial vertex, and $\widetilde{\Phi}\left(v_{0}^{\prime}\right)=\left(\mu, m\left(v^{k}\right)\right)$. Then $\widetilde{\Phi}$ takes values in $\mathscr{X} \times \mathbb{Z}_{\geq 0} \times$ $\left\{v^{k}, v^{k+1}\right\}$ on $J^{\prime}$.

\subsection{Pruning}

Consider our map

$$
\Phi=\Phi_{\lambda}: J_{\infty} \rightarrow \mathscr{X}
$$

(which depended on the choice of a fixed almost corner $\lambda$ ). We now "prune" our tree $J_{\infty}$ to produce a new tree $J_{\lambda}$. Consider the set

$$
K_{\lambda}:=\left\{\begin{array}{l|l}
v \in J_{\infty} & \begin{array}{c}
\text { there exists } v_{1}, \ldots, v_{m} \text { such that } \Phi\left(v_{1}\right) \notin \mathscr{X}_{++} \\
\text {and } v_{1} \rightarrow v_{2} \rightarrow \ldots \rightarrow v_{m}=v
\end{array}
\end{array}\right\}
$$

and define $J_{\lambda}$ to be the full subgraph with vertices $J_{\infty} \backslash K_{\lambda}$. (That is, we remove all branches from $J_{\infty}$ that contain elements which are mapped to weights which are not strictly dominant.) The restriction of $\Phi$ to $J_{\lambda}$ defines a map

$$
\Phi: J_{\lambda} \rightarrow \mathscr{X}_{++} .
$$

Restricting the extension $\widetilde{\Phi}$ (which depended on an additional choice of label $n\left(v^{k}\right)$ ) of $\Phi$ to $J_{\infty,++}$ yields a map:

$$
\widetilde{\Phi}=\widetilde{\Phi}_{\lambda, n\left(v^{k}\right)}: J_{\lambda} \rightarrow \mathscr{X}_{++} \times \mathcal{M}
$$

\subsection{The alternative construction}

For any positive integer $m$ let

$$
\lambda_{m}:=(\ell-1) m \varpi_{1}+\varpi_{2} \quad \text { and } \quad g_{m}:=\left(\lambda_{m},(2 m \ell-1)\left(v^{-1}\right)\right) .
$$

The previous constructions provide us with a rooted tree $J_{\lambda_{m}}$ and a map

$$
\widetilde{\Phi}_{g_{m}}: J_{\lambda_{m}} \rightarrow \mathscr{X}_{++} \times \mathcal{M} .
$$

Consider the union of multisets

$$
Z^{\prime}:=\bigcup_{m \geq 1}\left\{\widetilde{\Phi}_{g_{m}}(v) \mid v \in J_{\lambda_{m}}\right\}
$$

The following lemma implies that the above algorithm provides an alternative construction of $\widetilde{Z}$. 
Lemma 5.4. We have $\widetilde{Z}=Z^{\prime} \backslash\left\{g_{m} \mid m \in \mathbb{Z}_{\geq 0}\right\}$.

Proof (sketch). Fix $m \geq 0$ and consider $\widetilde{\Phi}:=\widetilde{\Phi}_{g_{m}}$ as defined above. Also, set $q=\left(\ell m \varpi_{1}, 0\left(v^{0}\right)\right)$ and consider the set $\Lambda_{q}$ defined as above. We claim that we have

$$
\Lambda_{q} \backslash\{q\}=\left\{\widetilde{\Phi}_{g_{m}}(v) \mid v \in J_{\ell m \varpi_{1}}\right\} \backslash\left\{g_{m}\right\},
$$

which implies the lemma. The equality (5.1) follows from the following local considerations:

1. The restriction of $\widetilde{\Phi}$ to the initial component of type $I$ takes the same values as part (1) of "Dynamics on walls", except for the first two values ( $g_{m}$ and $q$ respectively) which are removed in (5.1).

2. The restriction of $\widetilde{\Phi}$ to any component of type $I$ other than the initial segment takes the same values as those produced by part (2) of "Dynamics on the walls".

3. If $v$ (resp. $\left.v^{\prime}, v^{\prime \prime}\right)$ denotes the source (resp. sinks) of a component of type $J$ then $\widetilde{\Phi}\left(v^{\prime}\right)$ and $\widetilde{\Phi}\left(v^{\prime \prime}\right)$ are obtained from $\widetilde{\Phi}(v)$ by a giant leap, i.e., part (3) of "Dynamics on the walls".

4. For each component of type $J$ in $J_{\infty}$, if we consider the full subgraph

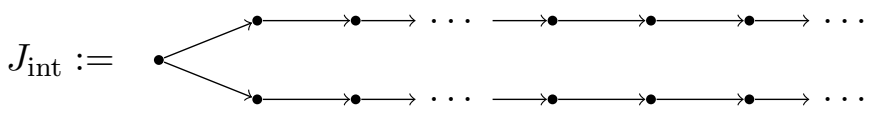

then the restriction of $\widetilde{\Phi}$ to each branch of $J_{\text {int }}$ produces the same set as the algorithm in Section 4.4 ("Billiards in an alcove").

\section{The conjecture}

Recall the notion of an $\ell$-alcove in $\mathscr{X}_{\mathbb{R}}$ from above. An alcove is a 1 -alcove. The alcove $\mathcal{A}_{0}:=$ $\left\{\lambda \mid 0<\left\langle\alpha^{\vee}, \lambda\right\rangle<1\right.$ for all $\left.\alpha^{\vee} \in \Phi_{+}^{\vee}\right\}$ is the fundamental alcove. The map $x \mapsto x \mathcal{A}_{0}$ gives a bijection between the affine Weyl group $\mathcal{W}$ and the set of alcoves. It restricts to a bijection between ${ }^{f} \mathcal{W}$ and the dominant alcoves.

Given $\mu \in \mathscr{X}$ the open box

$$
B_{\mu}:=\left\{\lambda \in \mathscr{X}_{\mathbb{R}} \mid\left\langle\alpha^{\vee}, \mu\right\rangle<\left\langle\alpha^{\vee}, \lambda\right\rangle<\left\langle\alpha^{\vee}, \mu\right\rangle+1 \text { for all } \alpha^{\vee} \in \Sigma^{\vee}\right\}
$$

contains exactly 2 alcoves. In this way we obtain a map $\mathcal{W} \rightarrow \mathscr{X}$ by sending $x \in \mathcal{W}$ to the unique $\mu \in \mathscr{X}$ such that $B_{\mu}$ contains $x \mathcal{A}_{0}$. It restricts to a map

$$
\kappa:{ }^{f} \mathcal{W} \rightarrow \mathscr{X}_{+} .
$$

Let $s_{0}$ denote the simple affine reflection. Consider the elements

$$
x_{0}:=\mathrm{id}, x_{1}=s_{0}, x_{2}=s_{0} s_{1}, x_{3}=s_{0} s_{1} s_{2}, x_{4}:=s_{0} s_{1} s_{2} s_{0}, \ldots
$$

of $\mathcal{W}$. (These are the alcoves along one edge of the dominant cone.) We have $\kappa\left(x_{2 i}\right)=\kappa\left(x_{2 i+1}\right)=$ $i \varpi_{1}$ for all $i \geq 0$.

We now describe a sort of inverse to $\kappa$. Recall that $\mathscr{X}_{++}=\mathbb{Z}_{>0} \varpi_{1} \oplus \mathbb{Z}_{>0} \varpi_{2}$ denotes the strictly dominant weights. Consider $\mu \in \mathscr{X}_{++}$and let $x, x^{\prime}$ denote the two elements of ${ }^{f} \mathcal{W}$ indexing alcoves contained in $B_{\mu}$. If $\mathcal{R}(z)=\{s \in \mathcal{S} \mid z s<z\}$ denotes the right descent set then it is easy to see that we have

$$
\mathcal{R}(x) \sqcup \mathcal{R}\left(x^{\prime}\right)=\left\{s_{0}, s_{1}, s_{2}\right\}=\mathcal{S} \quad \text { (disjoint union). }
$$


It follows that for any pair $\mu \in \mathscr{X}_{++}, s \in \mathcal{S}$ there is a unique element $x_{\mu}^{s} \in{ }^{f} \mathcal{W}$ such that $s \in \mathcal{R}\left(x_{\mu}^{s}\right)$ and $\mu=\kappa\left(x_{\mu}^{s}\right)$.

Recall our prime $p$ from above, and consider the multiset $\widetilde{Z}$ constructed in the previous section with $\ell=p$. We now describe how to use $\widetilde{Z}$ to define new elements in the anti-spherical module $\operatorname{AS}_{v}$. Consider the $\mathbb{Z}$-linear map $\varphi: \mathbb{Z}[v] \rightarrow \mathbb{Z}\left[v^{ \pm 1}\right]$ given by $v^{0} \mapsto 1$ and $v^{i} \mapsto v^{i}+v^{-i}$ for $i>0$. Set ${ }^{p} \zeta_{0}:=\underline{n}_{x_{0}}$. For any $i>0$ let $s \in \mathcal{S}$ denote the unique element of $\mathcal{R}\left(x_{i}\right)$ and consider the element:

$$
{ }^{p} \zeta_{i}:=\underline{n}_{x_{i}}+\sum_{\substack{\left(\mu, n\left(v^{k}\right)\right) \in \widetilde{Z} ; \\ n \in\{i, i-1, i-2\}}} \varphi\left(v^{k}\right) \underline{n}_{x_{\mu}^{s}} .
$$

Conjecture 6.1. We have:

1) ${ }^{p} \zeta_{i}={ }^{p} \underline{n}_{x_{i}}$ for $0 \leq i<2 p(p+1)$;

2) ${ }^{p} \zeta_{i}={ }^{p} \underline{n}_{x_{i}}^{2}$ for all $0 \leq i$.

Remark 6.2. Some remarks on the conjecture:

1. For an example of $\widetilde{Z}$ the reader is referred to Example 4.5.

2. It is a nice exercise to compare our conjecture to the results of J.G. Jensen [12] and Parker [20]. For example, in Fig. 1, the results of Jensen and Parker are explained by the unique alcoves labelled 12(1), 14(1), 16(1), 18(1) and 21(v).

3. Our conjecture (in particular the definition of the set $\widetilde{Z}$ ) does not seem to make sense for $p=2$.

4. We have verified part (1) of the conjecture for $p=3,5$ and in many cases for $p=7$ by computer. (In fact these calculations led to the conjecture.)

5. To determine the $p$-canonical basis in $\mathrm{AS}_{v}$ it is enough to know the elements ${ }^{p} \underline{n}_{x_{i}}$ for all $i>0$. (After exploiting the automorphism $s_{0} \mapsto s_{0}, s_{1} \mapsto s_{2}, s_{2} \mapsto s_{1}$ this can be deduced from a $v$-analogue of the fact that one can apply the tilting tensor product theorem to determine all tilting characters, provided one knows the tilting characters along the walls and in the $(p-1) \rho$-shift of the fundamental box, see [24, Section 1.6]. One can check by hand that one has

$$
{ }^{p} \underline{n}_{x}=\underline{n}_{x} \quad \text { for all } x \in\left\{\text { id, } s_{0}, s_{0} s_{1} s_{2} s_{1}, s_{0} s_{1} s_{2} s_{1} s_{0}\right\}
$$

and so the only remaining cases are ${ }^{p} \underline{n}_{x_{i}}$ for $i>1$.)

6. Similarly, to know all elements ${ }^{p} \underline{n}_{x}^{2}$ for $x \in{ }^{f} \mathcal{W}$ it should be enough to know ${ }^{p} \underline{n}_{x_{i}}^{2}$ for all $i$. Thus our conjecture gives a formula for the ${ }^{p} \underline{n}_{x}^{2}$ (which currently have no other rigorous definition $\left.^{4}\right)$.

7. Recall that the exists a bijection between two-sided cells in the affine Weyl group and nilpotent orbits in the dual group [16]. Moreover, every two sided cell intersects ${ }^{f} \mathcal{W}$ in a left cell (the canonical left cell) [19]. The "difficult" elements $x_{i}$ for $i>0$ all lie in the left cell corresponding to the minimal nilpotent orbit of $\mathrm{SL}_{3}$. This suggests that the problem of determining tilting characters should be related to the geometry of nilpotent orbits. Related results (connecting the $p$-canonical basis to coherent sheaves on the Springer resolution) may be found in $[3,4]$.

\footnotetext{
${ }^{4}$ However see the last sentence of Remark 2.1.
} 
8. After taking Remark 4.3 into account, our conjecture implies that the multiplicities of Weyl modules in the tilting module $T_{3 p k \varpi_{1}}$ grow (at least) exponentially in $k$. Thus our conjecture implies that decomposition numbers for symmetric groups $S_{n}$ grow exponentially in $n$ (see $[7,10]$ for the connection between tilting module characters and decomposition numbers), and that the dimension of the tilting module $T_{k \varpi_{1}}$ grows exponentially in $k$. Neither of these statements is true for $\mathrm{SL}_{2}$ or for the quantum group of $\mathrm{SL}_{3}$ at a $p^{\text {th }}$ root of unity.

9. The powers of $v$ which occur after $n$ iterations of the algorithm of Section 4.3 are all at least $n$. (By contrast, the algorithm Section 4.4 only ever changes the power of $v$ by \pm 1 .) This implies (assuming our conjecture) that ${ }^{p} \underline{n}_{x_{i}}$ involves arbitrarily high powers of $v$ as $i$ grows. This in turn implies that certain structure constants for the action of $\underline{h}_{s_{i}}$ on the $p$-canonical basis involve arbitrarily high powers of $v$. One can use this observation (and [21, Section 1.4]) to conclude that the analogue of the $a$-function for the $p$-canonical basis for the Hecke algebra $\mathrm{H}$ of $\mathcal{W}$ is unbounded.

10. Fix $m=\left(\mu, n\left(v^{k}\right)\right) \in \widetilde{Z}$ and let $x, x^{\prime}$ index the two alcoves contained in $B_{\mu}$, chosen such that $\mathcal{R}\left(x_{n}\right) \subset \mathcal{R}(x)$. The formula for ${ }^{p} \zeta_{i}$ above implies that $m$ contributes $\varphi\left(v^{k}\right)$ to the coefficient of $\underline{n}_{x}$ (resp. $\underline{n}_{x^{\prime}}, \underline{n}_{x}$ ) in ${ }^{p} \zeta_{n}$ (resp. ${ }^{p} \zeta_{n+1},{ }^{p} \zeta_{n+2}$ ). A key step in arriving at the multiset $\widetilde{Z}$ is to observe that the elements of the $p$-canonical basis $\left\{{ }^{p} \underline{n}_{x_{i}}\right\}$ may be decomposed into such "triples". After a first version of this paper was written, L.T. Jensen [13] has proved that such a decomposition is always possible, as a consequence of more general results on the $p$-canonical basis and the star operations of Kazhdan and the first author.

Finally, let us explain how to go from the $p$-canonical basis to a picture similar to that at the beginning of this paper (which is conjecturally described by $\widetilde{Z}$ ). It was this procedure (combined with heuristics as to what constitutes generation 2) that led us to our conjecture.

Fix an alcove $y \mathcal{A}_{0}$ (for $y \in{ }^{f} W$ ) which is not on a wall. For all $i \geq 0$ such that ${ }^{p} n_{y, x_{i}} \neq 0$, we consider the polynomial obtained from ${ }^{p} n_{y, x_{i}}$ by discarding negative powers of $v$, and write $i(f)$ in the alcove $y \mathcal{A}_{0}$. This produces a diagram, in which the strictly dominant alcoves are decorated by symbols of the form $i(f) .{ }^{5}$ Now, for all $\mu \in \mathscr{X}_{++}$we replace each "triple" of the form

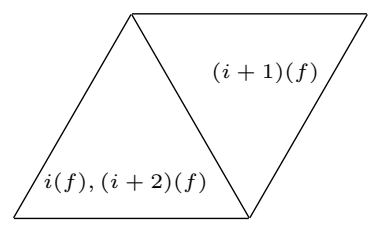

or

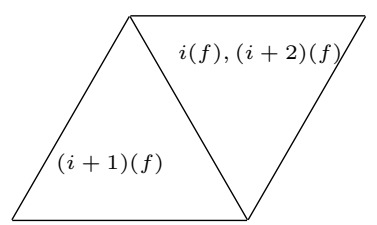

by

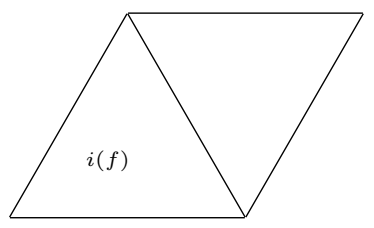

This is what is depicted (for a second generation version) in Fig. 1.

\section{Acknowledgements}

We would like to thank the anonymous referees for their comments.

\footnotetext{
${ }^{5}$ For an example of such a diagram for $p=5$ see http://www.maths.usyd.edu.au/u/geordie/pCanA2/ p5pretriples.pdf.
} 


\section{References}

[1] Achar P.N., Makisumi S., Riche S., Williamson G., Free-monodromic mixed tilting sheaves on flag varieties, arXiv:1703.05843.

[2] Achar P.N., Makisumi S., Riche S., Williamson G., Koszul duality for Kac-Moody groups and characters of tilting modules, arXiv:1706.00183.

[3] Achar P.N., Rider L., The affine Grassmannian and the Springer resolution in positive characteristic, Compos. Math. 152 (2016), 2627-2677, arXiv:1408.7050.

[4] Achar P.N., Rider L., Reductive groups, the loop Grassmannian, and the Springer resolution, arXiv:1602.04412.

[5] Andersen H.H., Filtrations and tilting modules, Ann. Sci. École Norm. Sup. (4) 30 (1997), 353-366.

[6] Angiono I.E., A quantum version of the algebra of distributions of $\mathrm{SL}_{2}$, Publ. Res. Inst. Math. Sci. 54 (2018), 141-161, arXiv:1607.04869.

[7] Donkin S., On tilting modules for algebraic groups, Math. Z. 212 (1993), 39-60.

[8] Elias B., Quantum Satake in type A. Part I, J. Comb. Algebra 1 (2017), 63-125, arXiv:1403.5570.

[9] Elias B., Losev I., Modular representation theory in type A via Soergel bimodules, arXiv:1701.00560.

[10] Erdmann K., Symmetric groups and quasi-hereditary algebras, in Finite-Dimensional Algebras and Related Topics (Ottawa, ON, 1992), NATO Adv. Sci. Inst. Ser. C Math. Phys. Sci., Vol. 424, Kluwer Acad. Publ., Dordrecht, 1994, 123-161.

[11] James G., The decomposition matrices of $\mathrm{GL}_{n}(q)$ for $n \leq 10$, Proc. London Math. Soc. 60 (1990), 225-265.

[12] Jensen J.G., On the character of some modular indecomposable tilting modules for $\mathrm{SL}_{3}$, J. Algebra 232 (2000), 397-419.

[13] Jensen L.T., p-Kazhdan-Lusztig theory, Ph.D. Thesis, Max Planck Institute for Mathematics, Bonn, 2017.

[14] Jensen L.T., Williamson G., The p-canonical basis for Hecke algebras, in Categorification and Higher Representation Theory, Contemp. Math., Vol. 683, Amer. Math. Soc., Providence, RI, 2017, 333-361, arXiv:1510.01556.

[15] Libedinsky N., Williamson G., The anti-spherical category, arXiv:1702.00459.

[16] Lusztig G., Cells in affine Weyl groups. IV, J. Fac. Sci. Univ. Tokyo Sect. IA Math. 36 (1989), $297-328$.

[17] Lusztig G., On the character of certain irreducible modular representations, Represent. Theory 19 (2015), 3-8, arXiv:1407.5346.

[18] Lusztig G., Williamson G., On the character of certain tilting modules, Sci. China Math. 61 (2018), 295-298, arXiv:1502.04904.

[19] Lusztig G., Xi N.H., Canonical left cells in affine Weyl groups, Adv. Math. 72 (1988), 284-288.

[20] Parker A., Some remarks on a result of Jensen and tilting modules for $\mathrm{SL}_{3}(k)$ and $q-\mathrm{GL}_{3}(k)$, arXiv:0809.2249.

[21] Riche S., Williamson G., Tilting modules and the $p$-canonical basis, arXiv:1512.08296.

[22] Soergel W., Charakterformeln für Kipp-Moduln über Kac-Moody-Algebren, Represent. Theory 1 (1997), $115-132$.

[23] Soergel W., Kazhdan-Lusztig polynomials and a combinatoric for tilting modules, Represent. Theory 1 (1997), 83-114.

[24] Williamson G., Algebraic representations and constructible sheaves, Jpn. J. Math. 12 (2017), 211-259, arXiv:1610.06261.

[25] Williamson G., Examples of $p$-canonical bases for the anti-spherical module for $\mathrm{SL}_{3}$, available at http: //www . maths. usyd. edu. au/u/geordie/pCanA2/.

[26] Williamson G., How to calculate many new decomposition numbers for symmetric groups, in preparation. 